\title{
INTELLIGENT CONTROL OF MAGNETIC LEVITATION SYSTEM
}

\author{
Ahmed H. Fares ${ }^{1}$, Amer abdelfattah ${ }^{2}$, \\ Abdel Badie Sharkawy ${ }^{3}$ and Ahmed A. Abo-Ismail ${ }^{4}$ \\ ${ }^{1}$ Researcher Assistant, ${ }^{2}$ Assistant Professor, ${ }^{3}$ Assistant Professor, ${ }^{4}$ Professor \\ ${ }^{1,3,4}$ Mechatronics Section, Department of Mechanical Engineering, \\ ${ }^{2}$ Electrical Engineering Department, Faculty of Engineering, \\ Assiut University, Assuit (71516), Egypt
}

(Received May 15, 2009 Accepted June 25, 2009).

This work investigates the design of a controller for keeping a steel ball suspended in the air. The main function of the controller is to maintain the balance between the magnetic force and the ball's weight. A PD fuzzy controller is designed for this unstable nonlinear system to maintain the balance between the magnetic force and the ball's weight and to achieve robust stability against model uncertainties.

KEYWORDS: Magnetic Levitation System, nonlinear system, intelligent control, fuzzy control

\section{INTRODUCTION}

Levitation is the stable equilibrium of an object without contact and can be achieved using electric or magnetic forces.[1,2] In a magnetic levitation, or maglev, system a ferromagnetic object is suspended in air using electromagnetic forces. These forces cancel the effect of gravity, effectively levitating the object and achieving stable equilibrium. In this paper, comparisons of control strategies are conducted. The controllers are analyzed for stability, robustness, and system response. It is representative of real-world applications of maglev technology in bearings and high speed transportation systems (Nagurka and Wang, 1997). In theory, the magnetic force produced by passing current through an electromagnet can exactly counteract the weight of the object. In practice, the electromagnetic force is sensitive to small disturbances that can induce acceleration forces on the levitated object, causing the object to be unbalanced. The main function of the controller is to maintain both static and dynamic equilibrium between the magnetic force and the object's weight, in the face of disturbances, using the input of the sensor to obtain the position of the object. Magnetic levitation offers a number of advantages. First, because parts do not contact, there is no wear [3]. Second, no lubrication is needed to prevent wear and reduce friction. This means that magnetic levitation approaches tend to be cleaner. Third, very high speeds are obtainable, as has been demonstrated with magnetic bearings.

Fourth, due primarily to the elimination of friction, one can obtain systems with minimum losses. Fifth, magnetic levitation systems can be made insensitive to hostile environments such as vacuum and steam. Magnetic levitation which has no contact between the moving part and fixed part is one of the good tools for a micromachine because mechanical friction disappears which increase the resolution and the 
accuracy of the positioning device, High speed trains Suspension in Japan and Germany, vibrations isolation systems, magnetic bearing, and superconductor rotor suspension of gyroscopes are good technological applications of this subject. Despite the fact that magnetic levitation systems have unstable behavior and are described by highly nonlinear differential equations, most designs approach are based on the linearzed models about a nominal operating point. In this case the tracking performance deteriorates rapidly with increasing deviation about the operating point, however, in order to ensure very long ranges of travel and still obtain good tracking; it is necessary to consider a nonlinear model rather that a linear one.

\section{SYSTEM MODELING}

The basic components of the maglev system include a sensor (infrared emitter-detector pair), an actuator (the electromagnet), and a controller. The sensor is a phototransistor with a resistor and can be modeled as a simple gain element. The sensor produces a voltage, $v s$, proportional to the object's position with a gain, $\beta$, which is linear around the operating point and can be determined experimentally (Green, 1997). A free-body diagram for a simple maglev system levitating an object of mass, $m$, is shown in Figure1.

The motion of the object is constrained to the vertical axis, and it is also assumed that the center of mass coincides exactly with the point of application of the electromagnetic forces $[6,7]$.

$$
v_{s}=\beta x
$$

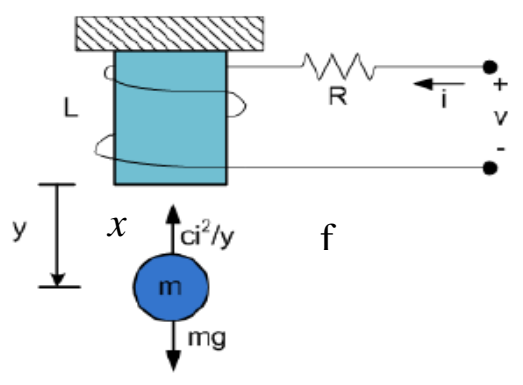

Figure 1: Free-body Diagram of a Levitated Object in Electromagnetic Levitation

The magnitude of the force $f(x, i, t)$ exerted across an air gap $x(t)$ by an electromagnet through which a current of magnitude $i(t)$ flows can be obtained using Faraday's inductive law and Ampere's circuit law as

$$
f(x, i, t)=-\frac{i(t)^{2}}{2} \frac{d L(x)}{d x}
$$

Where $\mathrm{L}(\mathrm{x})$ is the total inductance of the system

$$
L(x)=L_{1}+\frac{L_{O} x_{o}}{x_{0}}
$$


and $\mathrm{L}_{1}$ is the inductance of the coil in the absence of the levitated object, Lo is the additional inductance contributed by its presence and xo is the air gap when the levitated object is in equilibrium.

The inductance is characterized by the geometry and construction of the electromagnet, and can be determined experimentally as shown in figure 2. From (3), the derivative of inductance with respect to position is given by the following relation

$$
\frac{d L(x)}{d x}=-\frac{L_{0} x_{0}}{x^{2}}
$$

The relation between the coil inductance and the air gap is shown in Figure 2.

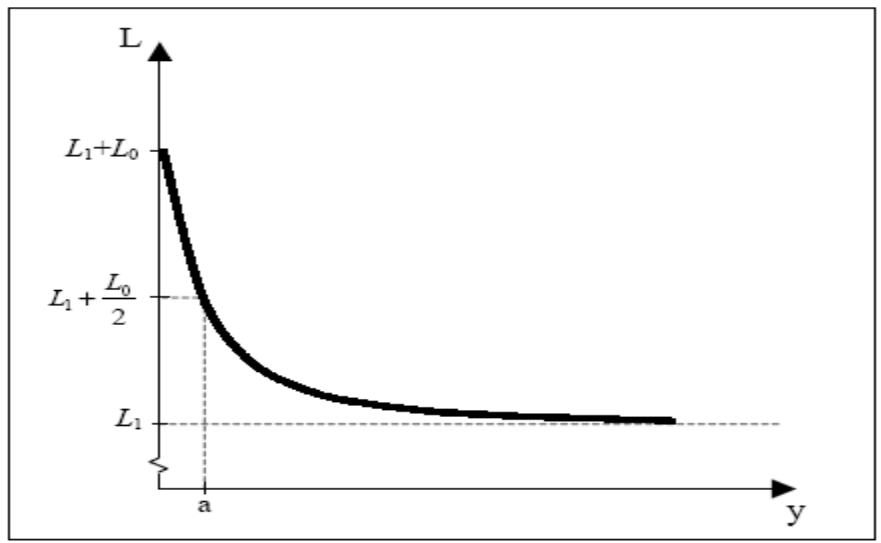

Figure 2: Relation between inductance and distance

Which, on combining with (2), gives

$$
f(x, i, t)=\frac{L_{O} x_{o}}{2}\left[\frac{i(t)}{x(t)}\right]^{2}=C\left[\frac{i(t)}{x(t)}\right]^{2}
$$

Where

$$
C=\frac{L_{O} x_{o}}{2}
$$

$\mathrm{C}$ is a constant that can be determined experimentally. Applying Newton's second law of motion and neglecting the effect of any drag forces, the governing equation of the system can be obtained as:

$$
m \frac{d^{2} x(t)}{d t^{2}}=m g-f(x, i, t)
$$

Substituting Equation (5) into Equation (7) gives:

$$
m \frac{d^{2} x(t)}{d t^{2}}=m g-C\left[\frac{i(t)}{x(t)}\right]^{2}
$$


Where $\mathrm{g}$ is the acceleration due to gravity. For designing a linear control strategy, the non-linear electromagnetic force in Equation (5) is linearzed about an equilibrium point $x_{0}[6,7]$.

$$
f(x, i, t)=C\left[\frac{i_{o}}{x_{o}}\right]^{2}+\left[\frac{2 C i_{0}}{x_{o}^{2}}\right] i(t)-\left[\frac{2 C i_{o}^{2}}{x_{o}^{3}}\right] x(t)+\ldots \ldots .
$$

$\mathrm{Or}$

$$
f(x, i, t)=f_{o}+f_{1}+\ldots \ldots \ldots
$$

Where

$$
i=i_{o}+\delta i \quad \& \quad x=x_{o}+\delta x
$$

Where $i_{0}$ and $x_{0}$ are the equilibrium values and $\delta \mathrm{i}$ and $\delta \mathrm{x}$ are incremental values for the current and position variables, respectively. In the following analysis, $i$ and $\mathrm{x}$ represent only the changes $(\delta \mathrm{i}$ and $\delta \mathrm{x}$ ) from equilibrium values of current and position, respectively, and not their absolute values. From the free body diagram in Fig. 1, at equilibrium the magnetic force on the levitated object equals the gravitational force. By defining $f_{0}$ as the force to balance the weight of the object at equilibrium

$$
f_{o}=C\left[\frac{i_{o}}{x_{o}}\right]^{2}
$$

Neglecting the higher order terms in Equation (9) and Equation(10), the incremental (control) force required for maintaining equilibrium $f(x, i, t)$ is

$$
f(x, i, t)=\left[\frac{2 C i_{o}}{x_{o}^{2}}\right] i(t)-\left[\frac{2 C i_{o}^{2}}{x_{o}^{3}}\right] x(t)
$$

The governing equation for the levitated object is determined by application of Newton's second law

$$
m \frac{d^{2} x(t)}{d t^{2}}=m g-f
$$

On combination with Equation (10), Equation (13), and Equation (14), Equation (15) gives

$$
m \frac{d^{2} x(t)}{d t^{2}}=m g-f_{0}-\left[\frac{2 C i_{o}}{x_{o}^{2}}\right] i(t)+\left[\frac{2 C i_{o}^{2}}{x_{o}^{3}}\right] x(t)
$$

Since at equilibrium, Equation (13) applies, Equation (16) becomes

$$
m \frac{d^{2} x(t)}{d t^{2}}=-\left[\frac{2 C i_{o}}{x_{o}^{2}}\right] i(t)+\left[\frac{2 C i_{o}^{2}}{x_{o}^{3}}\right] x(t)
$$

Taking the Laplace transform of the above equation gives

$$
m s^{2} X(s)=-\left[\frac{2 C i_{o}}{x_{o}^{2}}\right] I(s)+\left[\frac{2 C i_{o}^{2}}{x_{o}^{3}}\right] X(s)
$$


Thus, the transfer function of the system with the change in current to the coil as the input and the change in position of the levitated object as the output is given by

$$
G(S)=\frac{X(s)}{I(s)}=\frac{-\left[\frac{2 C i_{o}}{x_{o}^{2}}\right]}{s^{2} m-\left[\frac{2 C i_{o}^{2}}{x_{o}^{3}}\right]}=\frac{-\left[\frac{2 C i_{o}}{m x_{o}^{2}}\right]}{s^{2}-\left[\frac{2 C i_{o}^{2}}{m x_{o}^{3}}\right]}
$$

The transfer function has two poles, one of which is in the right half plane at $\sqrt{2 C i_{o}^{2}} / m x_{o}^{3}$ which makes it unstable in open-loop. A representation of the maglev system is shown in the block diagram in Figure 3.

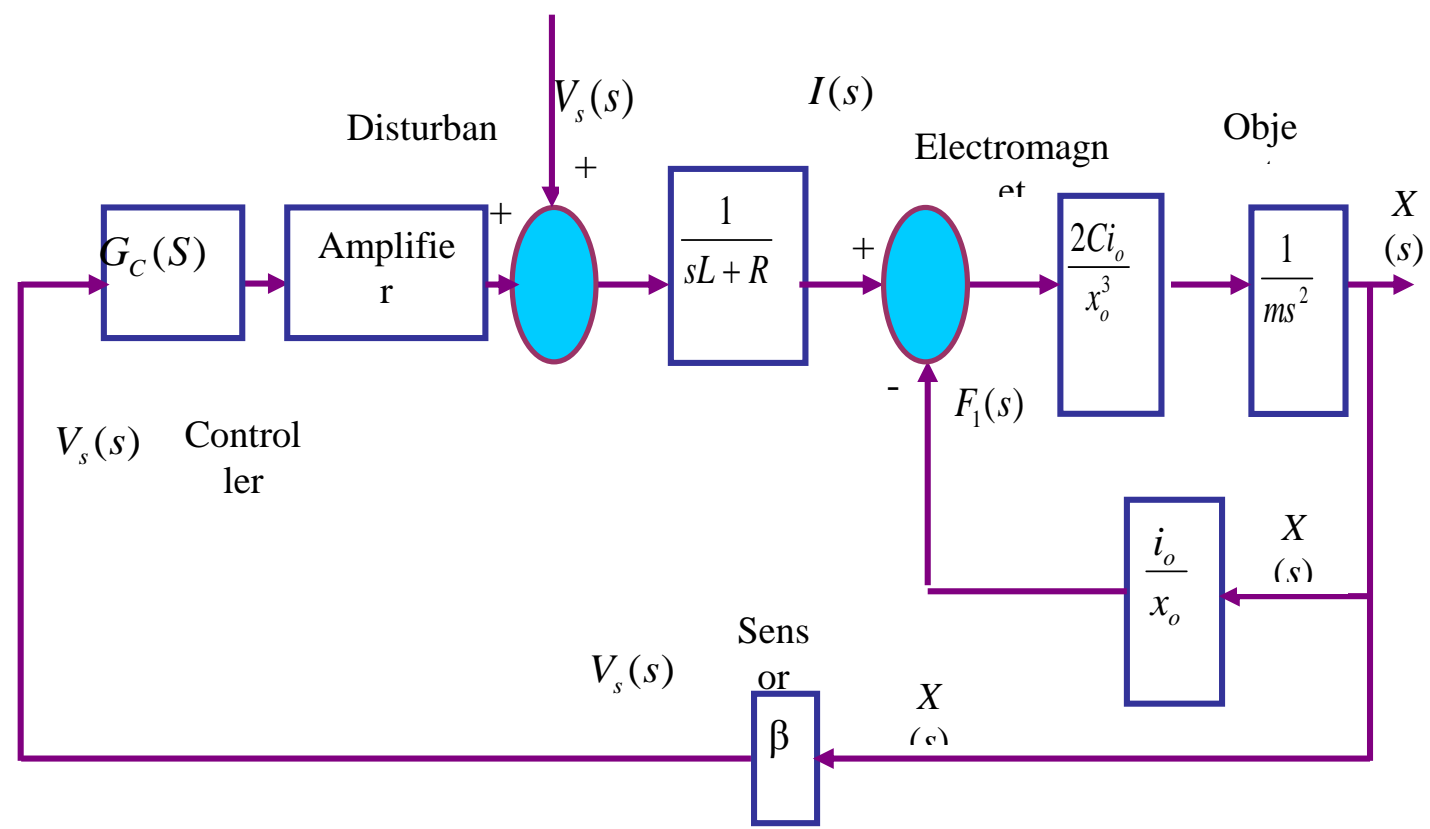

Figure 3: Block Diagram of the Closed-Loop Maglev System

The force constant, C, can be obtained from equation (13) experimentally by levitating an object of known mass, $\mathrm{m}$, and measuring the current, $i_{0}$ and position, $x_{0}$. The value of $L_{0}$ can then be calculated from Equation (6).Another way to interpret the transfer function for the maglev system is to consider the sensor output as the system output and the voltage to the electromagnet as the system input. The electromagnet can be represented as a series combination of a resistor and inductor. From Kirchoff's voltage law, the voltage, $\mathrm{v}$, in the coil can be determined as,

$$
v=R i(t)+L(x) \frac{d i(t)}{d t}
$$

Combining the Laplace transforms of the above equations, the overall transfer function between the voltage of the electromagnet as input and the sensor voltage as the output is determined as 


$$
G_{O}(s)=\frac{V_{S}(s)}{V(s)}=\frac{-2 \beta C i_{o} / m L x_{o}^{2}}{(s+R / L)\left(s^{2}-2 C i_{o}^{2} / m x_{o}^{3}\right)}
$$

Following extensive system identification, the overall transfer function between the sensor output and voltage input to the coil was found to be

$$
\mathrm{G}(\mathrm{S})=\frac{24.43 * 10^{3} G_{C}(S)}{(S+62.6)(S-62.6)(S+43)}
$$

The transfer function shows that the system has no zeros and a pole in the right half plane which makes it open-loop unstable.

A representation of the maglev system setup is shown in Figure 4.

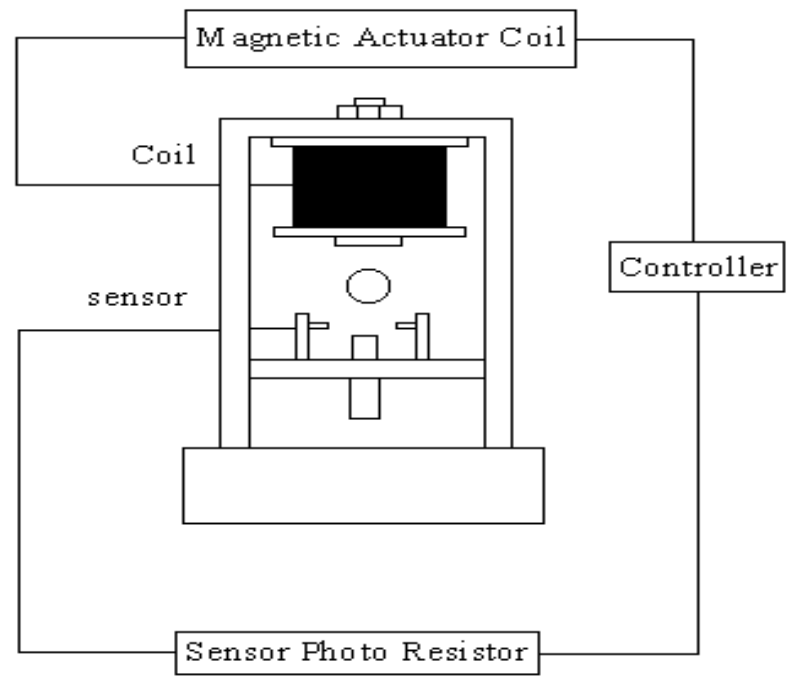

Figure 4: Schematic view of Magnetic levitation system layout

The sensor is a photo resistor and can be modeled as a simple gain element. The sensor produces a voltage, $V s$, proportional to the object's position, $x$, with a gain, $\beta$, which is linear around the operating point and can be determined experimentally as given in figure 5. Coil inductance is measured experimentally by measuring the time constant, the signal appear on the oscilloscope as shown in figure 6.The physical system interface wiring diagram is shown in figure 7 , the programming language used in interfacing physical system with the controller is BORLAND C 


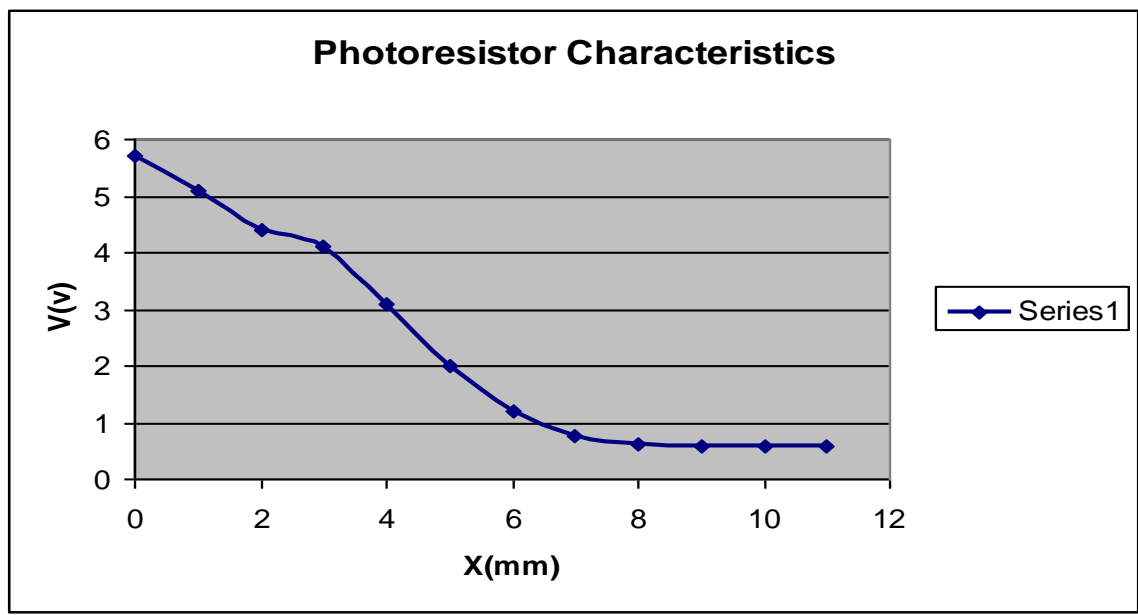

Figure 5: the characteristic of the optical contact less measurement system (Experimental)

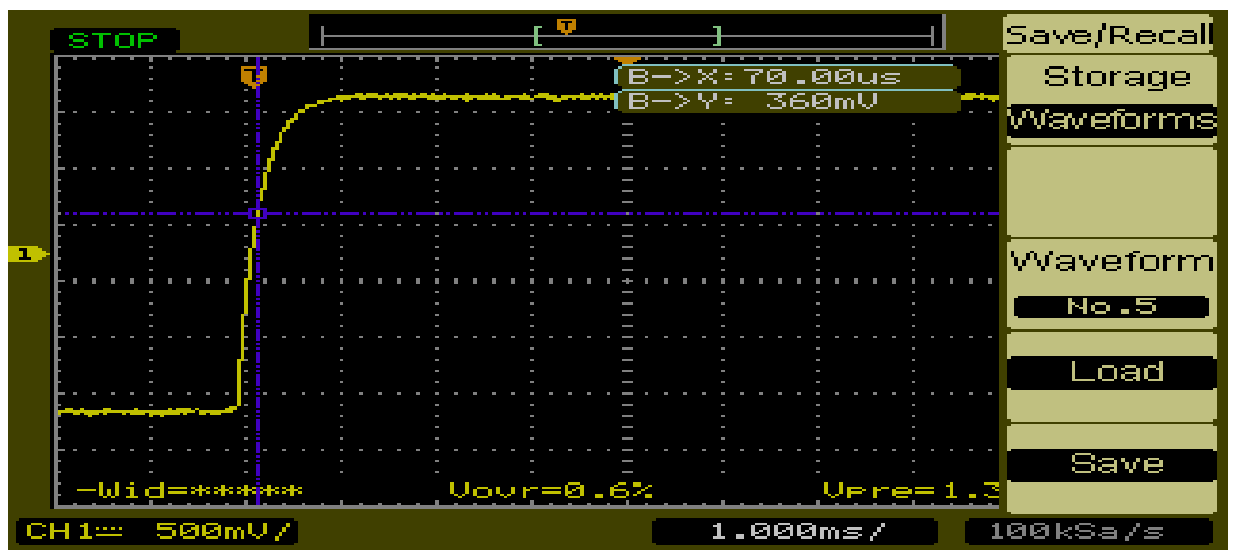

Figure 6: assignment of the coil inductance L (Experimental)

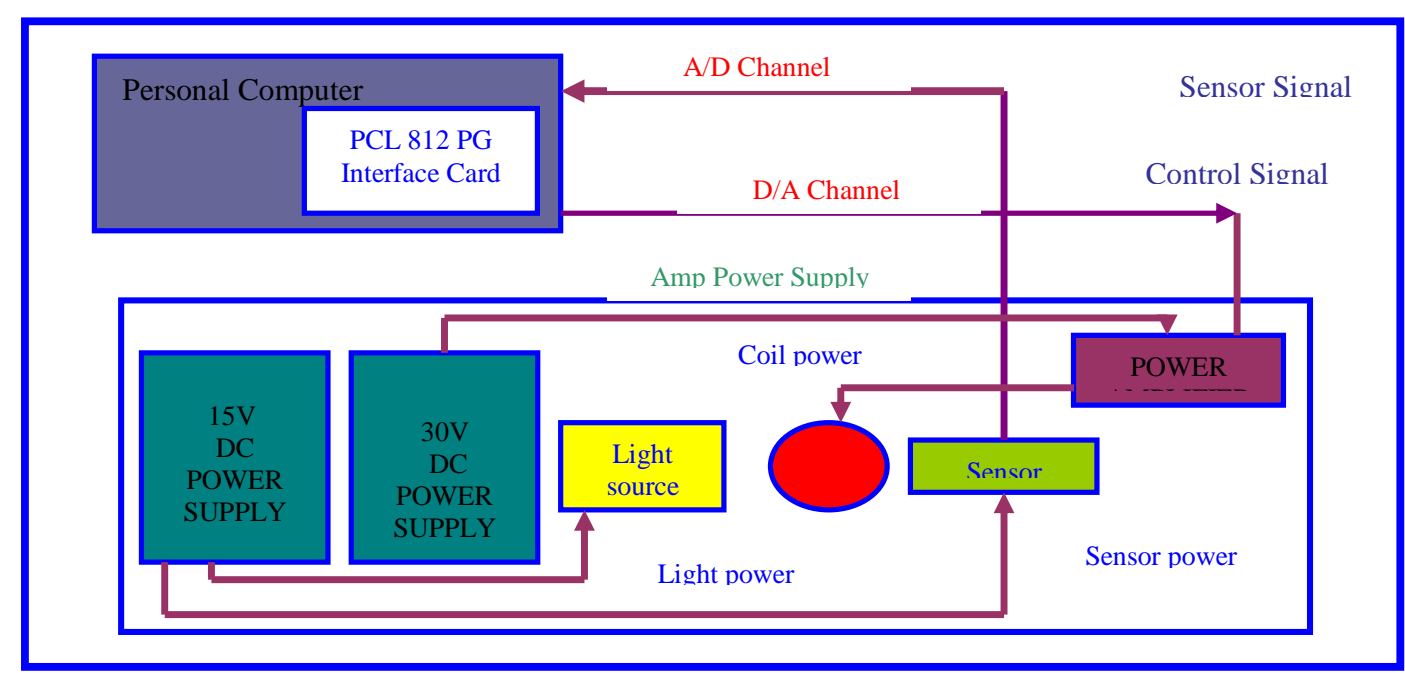

Figure 7: Magnetic Levitation System Interface 
The system physical parameters are given in Table (1)

Table (1) physical System Parameters

\begin{tabular}{|c|c|c|}
\hline parameter & Definition & Value \\
\hline $\mathbf{m}$ & Ball Mass & $\mathbf{0 . 0 7 k g}$ \\
\hline $\mathbf{R}$ & Coil Resistance & $\mathbf{3 4} \mathbf{~ o h m}$ \\
\hline $\mathbf{L}$ & Coil Inductance & $\mathbf{0 . 8 3 6} \mathbf{H}$ \\
\hline $\mathbf{N}$ & $\begin{array}{c}\text { Number Of Coil } \\
\text { Turnc }\end{array}$ & $\mathbf{4 1 9 0}$ Turns \\
\hline $\mathbf{C}$ & Constant & $\mathbf{0 . 0 0 0 1 4 7 6} \mathbf{~ N m} 2 / \mathbf{A 2}$ \\
\hline $\mathbf{K}$ & Constant & $\mathbf{4 . 0 2}$ N/A \\
\hline $\boldsymbol{\beta}$ & Sensor Gain & $\mathbf{3 5 5}$ V/A \\
\hline X0 & Nominal Airgap & $\mathbf{5} \mathbf{~ m m}$ \\
\hline I0 & Nominal Current & $\mathbf{0 . 3 4 1 A}$ \\
\hline
\end{tabular}

\section{CONTROLLER DESIGN}

\subsection{Phase Lead Compensated Controller}

The system is designed to carry out the major Function of stabilizing the working point of the levitation system. [4, 8] Equation (18) shows that this system has one stable pole, while the other is still unstable. The compensated root-locus is shown in Figure 8. This plot shows that the uncompensated system is unstable and cannot be stabilized simply by changing the system gain. The simplest way to stabilize the system is to use the phase-lead compensated controller to cancel the unstable pole. In order to pull the root-locus into the left-hand plane, a zero needs to be added to the phase-lead compensated controller in the left-hand plane between the first left-hand plane pole and the origin. The necessary pole required for the phase-lead compensated controller is placed deeper into the left-hand plane. This will minimize the impact of the pole of the compensated controller on the root-locus. The transfer function of the phase-lead compensated controller is shown as

$$
G_{c}(s)=K_{C} \frac{S+Z}{S+P}
$$

It seems reasonable to attempt pole-zero cancellation by placing the compensator zero at the system pole of $\mathbf{- 6 2 . 6}$ Choose $\mathrm{Z} 1=-62.6$ and $\mathrm{Z} 2=-43$ to compensate the open loop poles (-62.6). And we find that the pole of the compensator is to be $\mathrm{P} 1=-280$ and $\mathrm{P} 2=-138$ then the open loop transfer function of the system will be

$$
G_{C}(s) G(s)=\frac{6272000}{(S-62.6) *(S+138) *(S+280)}
$$




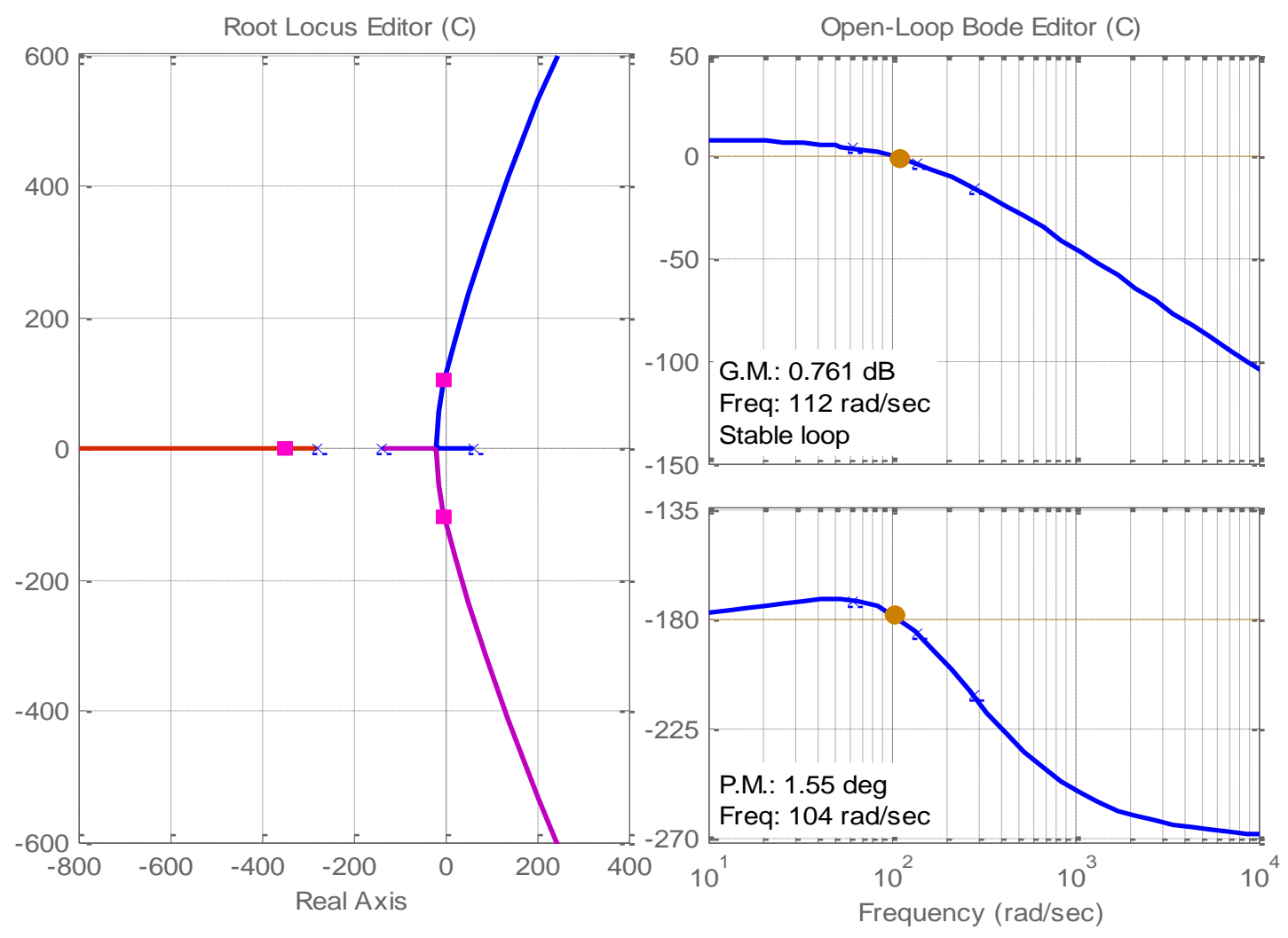

Figure 8: Root locus and the frequency response of the compensated system of the magnetic levitation system

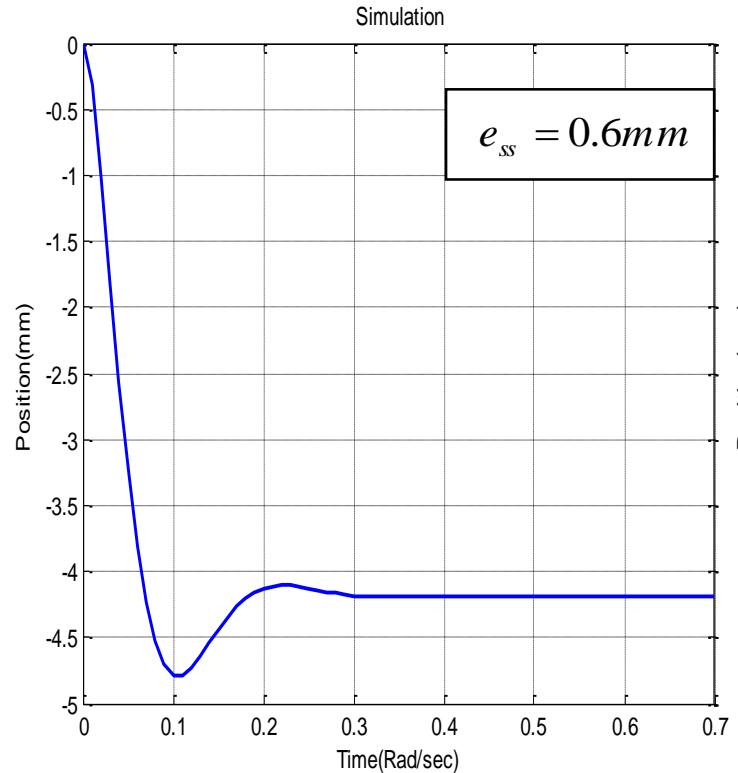

Figure 9: System responses for step disturbance

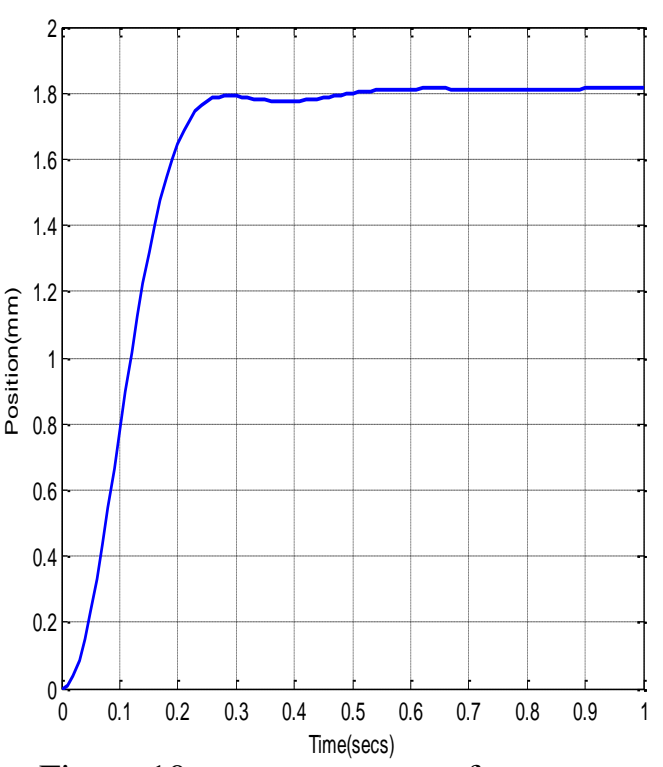

Figure 10: system response for a step disturbance force equivalent to $20 \%$ of the ball mass 
From the above simulation results of the phase lead compensated controller it is evident that the phase-lead controller can pull the uncompensated root locus in the right-hand plane into the left-hand plane, which indicates that the system can be stabilized as shown in Figure 8. In the transient state, the simulated result shows that the controller will cause the ball to return to its original position whenever it is disturbed. However, there is a steady state error due to the step noise of $e_{s s}=0.6 \mathrm{~mm}$. Obviously, changes in the electromagnet's current occur more quickly than variations in the ball's position, which indicates that the controlled electromagnet current can stabilize the disturbances that otherwise, would cause the ball to either fall or attach itself to the electromagnet.

\subsection{Proportional plus Derivative (PD) Controller:}

The following design requirements are chosen to obtain fast response and good damping characteristics: Settling time ts $=0.25 \mathrm{sec}$ and damping ratio $\xi=0.5$.

$$
G(S)=\frac{\mathrm{K}(\mathrm{S}+\mathrm{Z})}{(\mathrm{S}+62.6)(\mathrm{S}-62.6)(\mathrm{S}+43)}
$$

Then the compensated open loop transfer function will be

$$
G(s) G_{c}(s)=\frac{4733.5(\mathrm{~S}+34.5)}{(\mathrm{S}+62.6)(\mathrm{S}-62.6)(\mathrm{S}+43)}
$$

The simulated step response with the PD controller is obtained as shown in Figure (11) and Figure (12).

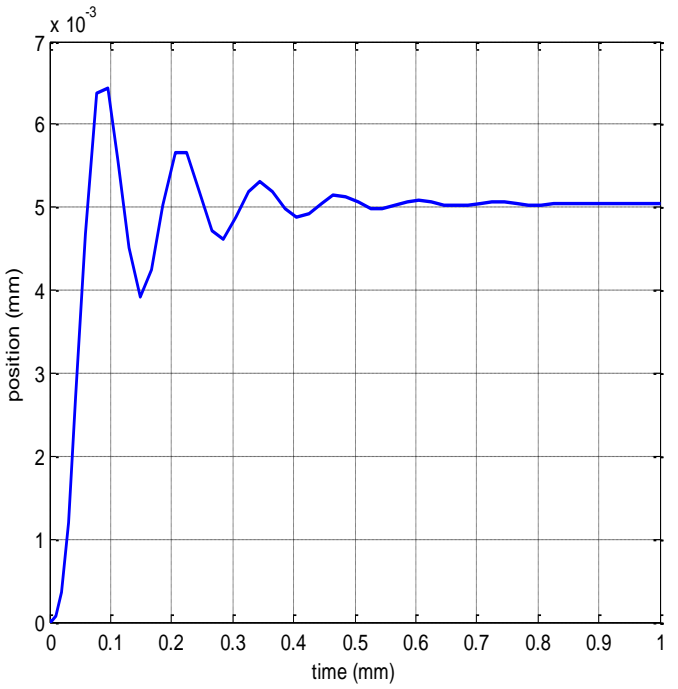

Figure 11: system response for $T_{d}=0.045$ and $K_{p}=3.28$

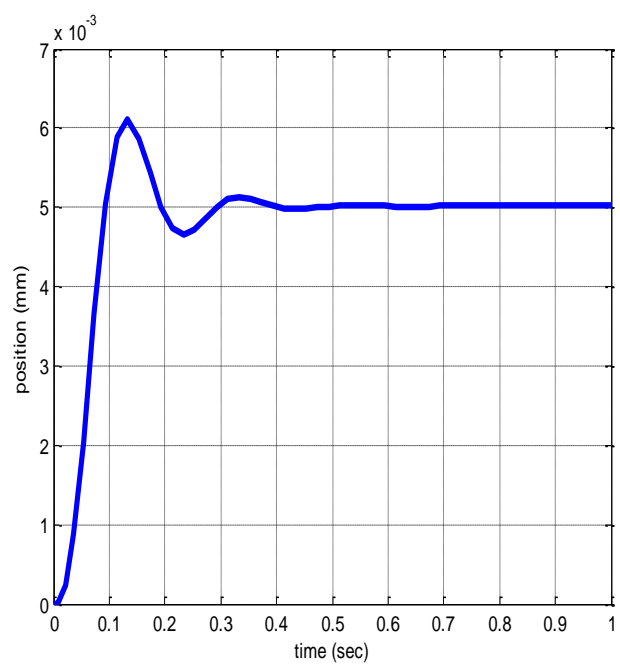

Figure 12: system response for $T_{d}=0.09$ and $K_{p}=5.1$ 
The parameters of the proportional plus derivative PD controller were tuned using the stability range program software.

The compensated root locus is shown in Figure 13 and simulink model of PD controller is shown in Figure 14 .

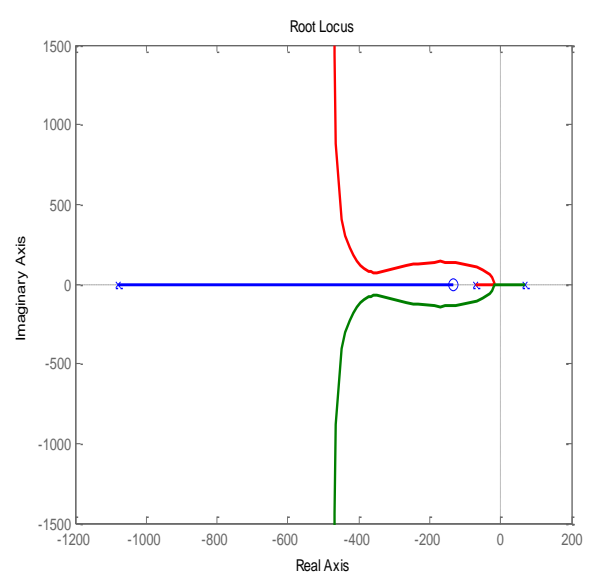

Figure 13: Root locus of the compensated system

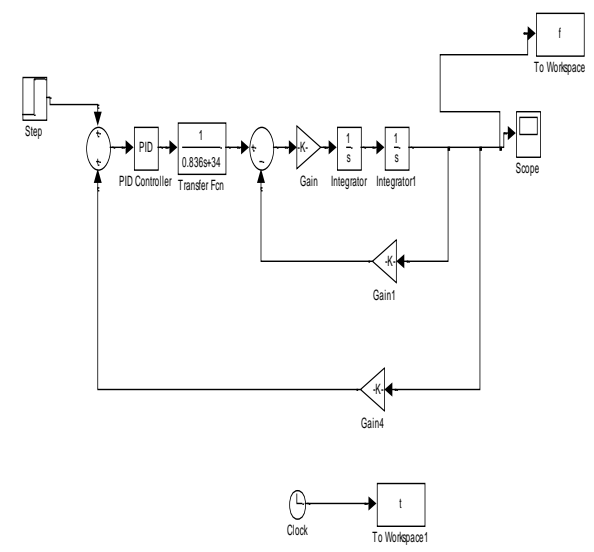

Figure 14: simulink model of conventional PD controller

\subsection{Fuzzy PD Controller}

The next step in the design procedure is to replace the phase-lead controller with an equivalent fuzzy system acting the same way $[10,11,12,13]$. Since the control problem requires a PD-type controller, choosing the error e $(\mathrm{kT})$ between the set point and the controlled value, and the change-in-error $\mathrm{c}(\mathrm{kT})=(\mathrm{e}(\mathrm{kT})-(\mathrm{e}(\mathrm{kT}-\mathrm{T})) / \mathrm{T}$ (derivative of the error) as inputs for the fuzzy system comes very natural.Generally, a fuzzy controller presents itself as a nonlinear input-output mapping, with three sources of nonlinearity: the rule base, the inference mechanism and the defuzzification method.It is possible to create a linear fuzzy control structure which acts like the summation of the PD controller, by adequately modeling the system's components. The resulting fuzzy system will act the same way as the initial PD controller.In order to achieve this, the following design choices are recommended:

- use of symmetric triangular membership functions, overlapped at 50\%;

- $\quad$ the and connective is associated with the algebraic product;

- $\quad$ use of singleton membership functions for the output variable;

- use of the center of gravity (COG) defuzzification method to determine the output $\Delta \mathrm{u}(\mathrm{kT})$

The elements of the system were designed according to the specification mentioned above. For each of the input variables seven membership functions were used (meaning up to $7^{\wedge} 2=49$ rules in the rule base). These were symmetric, $50 \%$ overlapping triangular shaped membership functions. Figure 15 shows the choice for the controller's membership functions $[14,15,16]$. 


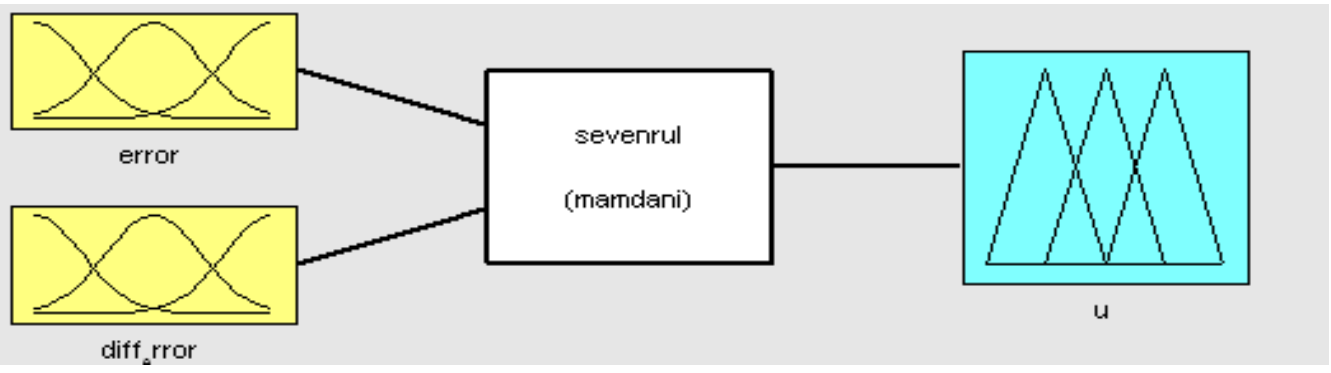

(a)

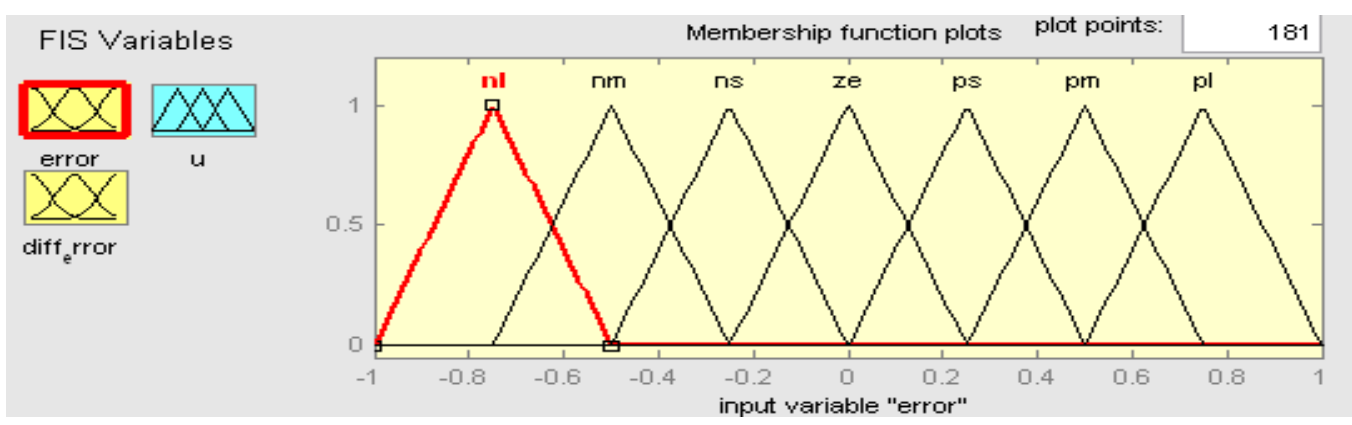

(b)

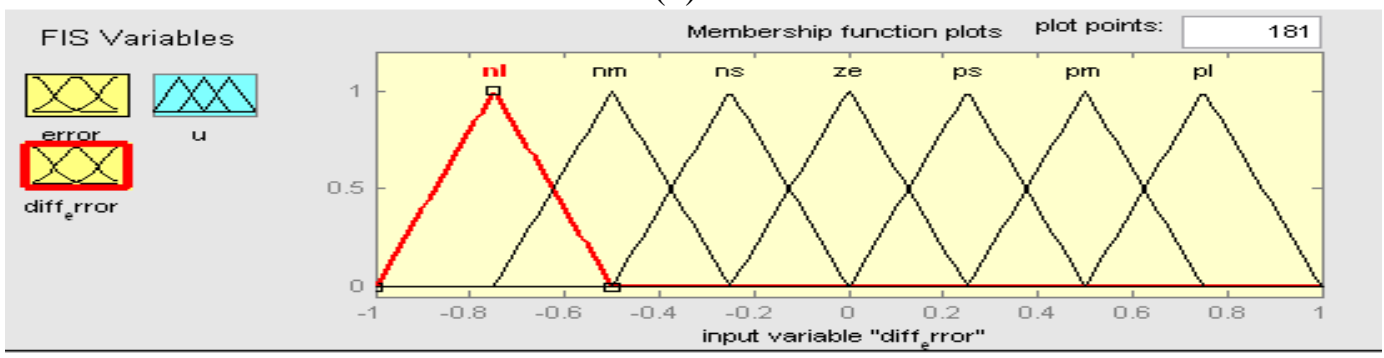

(c)

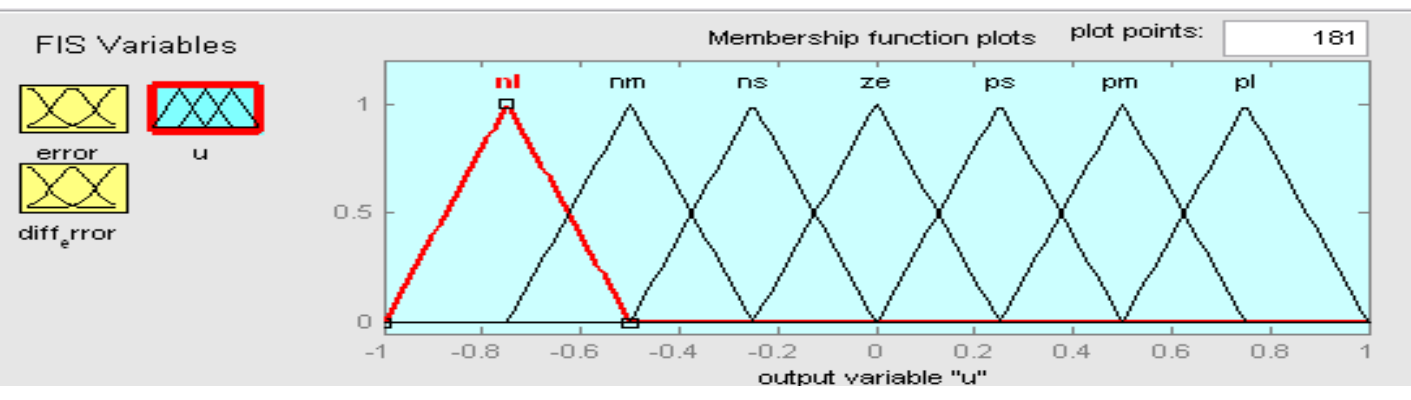

(d)

Figure 15: Membership functions for the fuzzy controller (a) Fuzzy PD system, (b) Input1(error),(c)Input 2(derivative of error), and (d) Output 
Following the design strategy mentioned in the previous paragraphs a fuzzy controller was developed. Its structure is presented as shown in figure 16.

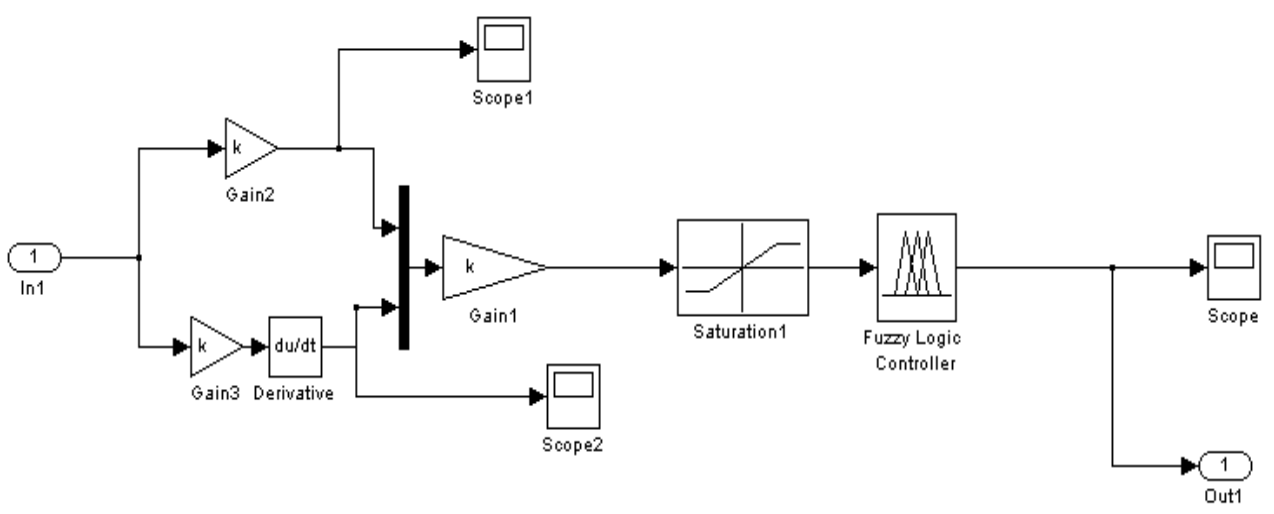

Figure 16: simulink model of Fuzzy PD controller

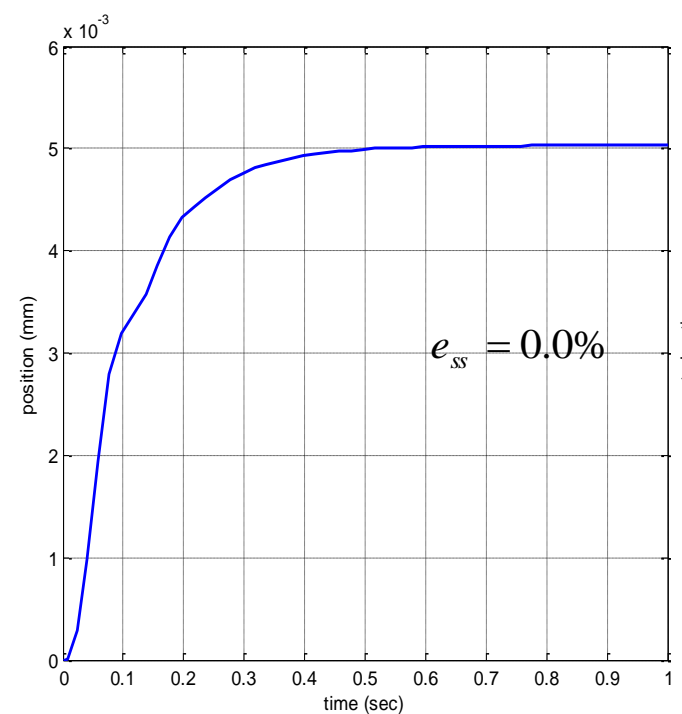

Figure 17: step response

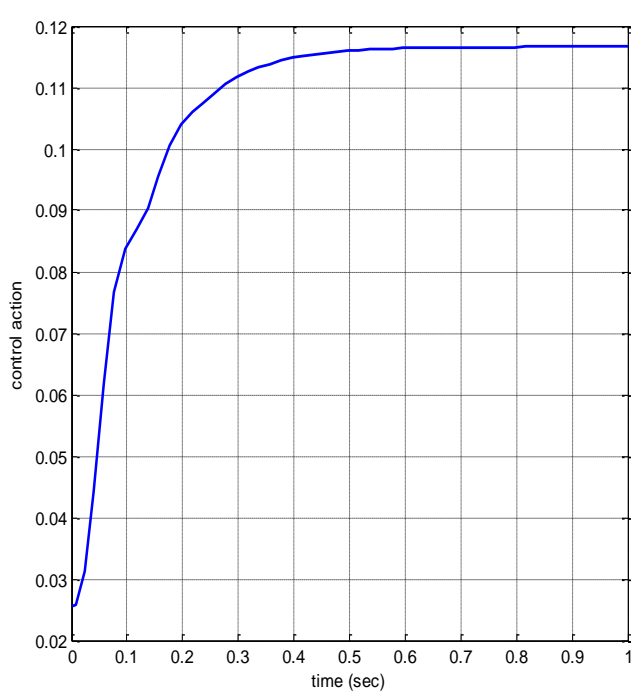

Figure 18: Fuzzy PD control action response 


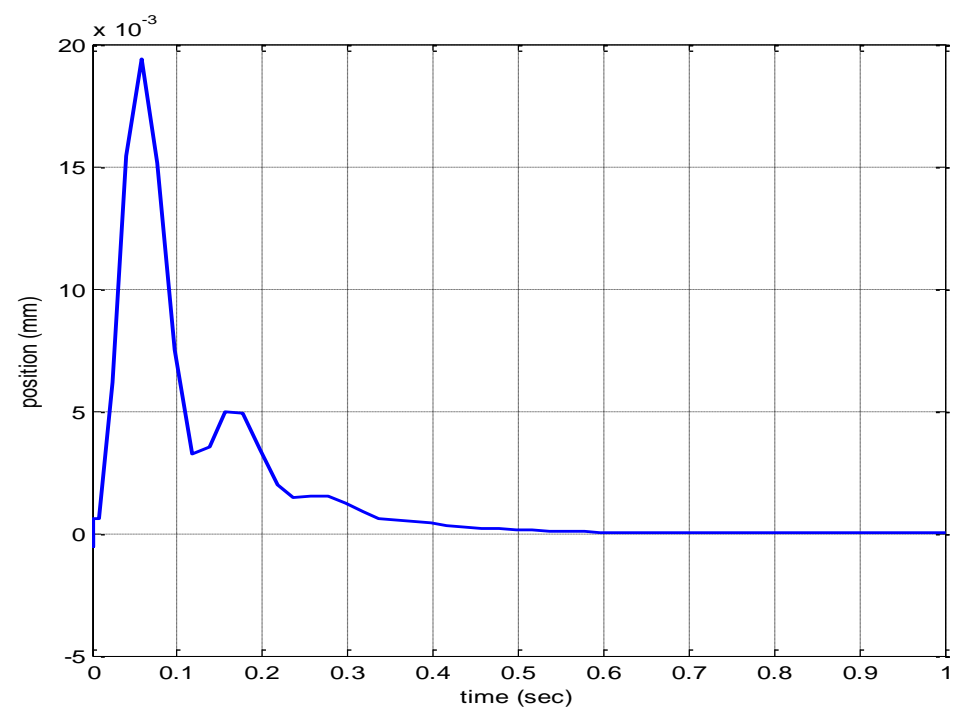

Figure 19: error signal response

It is clearly indicated from simulation results that the fuzzy PD controller have improved the magnetic levitation stability more than classical PD upon comparing the corresponding results of figure 17 and figure 18.

\section{CONCLUSIONS}

An approach for controlling the magnetic suspension system based on classical and fuzzy logic has been presented. The physical model of the magnetic suspension has been realized using the simple electromagnet and a steel ball. The simplified mathematical model has been developed and the parameters for the PD controller have been designed.. When a deviation of iron sphere from its original fixed position by external effects occurs, fuzzy logic control brings the iron sphere to its original position quicker than PD controller.

\section{REFERENCES}

1. S. Earnshaw, "On the nature of the molecular forces which regulate the constitution of the luminiferous ether", Trans. Camb. Phil. Soc., 7, 97-112 (1842).

2. Abdelfatah, M., A Tutorial on Magnetic Levitation and its Control System Design, Micro-Automation Group TR\#20, Mechanical Department, the University of Texas at Austin. Jan. 1992.

3. A.T.A. Peijnenburg, J.P.M. Vermeulen, J. Van Eijk, Magnetic Levitation Systems Compared to Conventional Bearing Systems, Journal of Microelectronic Engineering 83 (2006),pp. 1372-1375. 
4. YING-SHING SHIAO, Design and Implementation of a Controller for a Magnetic Levitation System, Proc.Natl. Sci. Counc.Roc (D), Vol. 11, No. 2, 2001, pp. 88-94.

5. Amer Abd El-Fattah, "Analysis and Design of Magnetic Levitation Control Systems", Master of Science Thesis, Assiut University, 1994.

6. Trumper, D.L.; Olson, S.M.; Subrahmanyan, P.K." Linear zing control of magnetic suspension systems" Control Systems Technology, IEEE Transactions on Volume 5, Issue 4, Jul 1997 Page(s):427 - 438.

7. B V Jayawant "Electromagnetic suspension and levitation"1981 Rep. Prog. Phys. 44 411-477.

8. Jianxin Tang, Alfred University. "Magnetic Levitation Systems Using Analog and Digital Phase-Lead Controllers" American Society for Engineering Education, 2007.

9. Mizuno, T. and Higuchi, T., Design of the control system of totally active magnetic bearings, in Proc. Inter-national Symposium on Design and Synthesis, Tokyo, Japan, 1984, pp. 534-539.

10. P.T. Chan, W.F. Xie, and A.B. Rad, "Tuning Of Fuzzy Controller for an OpenLoop Unstable System: A Genetic Approach", Fuzzy Sets and Systems, Elsevier Science, Vol. 111, Issue 2, pp. 137-152, April 2000.

11. Dale W. Van Cleave and K. S. Rattan, "Tuning of Proportional Plus Derivative Fuzzy Logic Controller for Nonlinear System Control", Joint $9^{\text {th }}$ IFSA World Congress and $20^{\text {th }}$ NAFIPS International Conference, Vol. 3, pp. $1288-1293$, July 2001

12. Basil Hamed, "Comparison of Fuzzy Logic and Classical Controller Designs for Nonlinear Systems", Doctor of Philosophy Dissertation, New Mexico State University, USA, 1999.

13. Hajjaji AE, OuladsineM.Modeling and nonlinear control of magnetic levitation system. IEEE Trans Indust Electr 2001;48:831-8.

14. B. Butkiewicz, "About Robustness of Fuzzy Logic PD and PID Controller under Changes of Reasoning Methods", ESIT 2000

15. Orhan, A., Akin, E., Karaköse, M. - DSP Implementation of Fuzzy Controlled Magnetic Levitation System, Fuzzy Days 2001, LNCS 2206, pp. 950-958.

16. Duka, A.V., Grif, H.Şt., Oltean S.E. - Fuzzy Control of an Electromagnetic Levitation Device, 13th International Symposium on Modeling, Simulation and System's Identification (SIMSIS-13), Galaţi, Romania, 2007 
"التحكم الأكي في منظومة رفع كهرومغناطيسي"

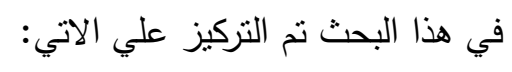

بعد بناء نموذج للنظام Model تم تصميم متحكمات تقليدية لضبط اتزان النظام منل

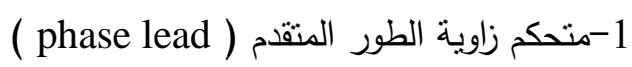

2- متحكم التتاسب والتفاضل (PD)

وتم مقارنة هذه المتحكمات التقليدية بمتحكمات ذكية مثل المتجكمات الفازية ( Fuzzy Logic)

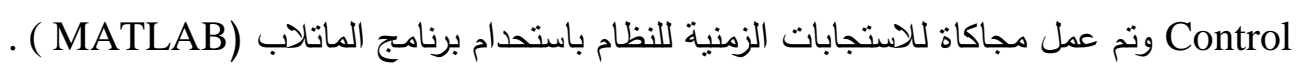

